\title{
Global well-posedness and multi-tone solutions of a class of nonlinear nonlocal cochlear models in hearing
}

\author{
Jack Xin* and Yingyong Qi ${ }^{\dagger}$
}

\begin{abstract}
We study a class of nonlinear nonlocal cochlear models of the transmission line type, describing the motion of basilar membrane (BM) in the cochlea. They are damped dispersive partial differential equations (PDEs) driven by time dependent boundary forcing due to the input sounds. The global well-posedness in time follows from energy estimates. Uniform bounds of solutions hold in case of bounded nonlinear damping. When the input sounds are multi-frequency tones, and the nonlinearity in the PDEs is cubic, we construct smooth quasi-periodic solutions (multi-tone solutions) in the weakly nonlinear regime, where new frequencies are generated due to nonlinear interaction. When the input is two tones at frequencies $f_{1}, f_{2}\left(f_{1}<f_{2}\right)$, and high enough intensities, numerical results illustrate the formation of combination tones at $2 f_{1}-f_{2}$ and $2 f_{2}-f_{1}$, in agreement with hearing experiments. We visualize the frequency content of solutions through the FFT power spectral density of displacement at selected spatial locations on BM.
\end{abstract}

\footnotetext{
${ }^{*}$ Department of Mathematics and TICAM, University of Texas at Austin, Austin, TX 78712, USA. Corresponding author, email: jxin@math.utexas.edu. This work was partially supported by ARO grant DAAD 19-00-1-0524 and NSF ITR-0219004.

${ }^{\dagger}$ Qualcomm Inc, 5775 Morehouse Drive, San Diego, CA 92121, USA.
} 


\section{Introduction}

Digital signal processing on sounds is an essential component of modern hearing devices [16], and a useful tool for evaluating acoustic theories of peripheral auditory systems, [13] among others. A fundamental issue is to model the auditory response to complex tones because the nonlinear interaction of acoustic waves of different frequencies allows for audio compression [16] among other applications. Nonlinearities are known to originate in the cochlea and are further modified in higher level auditory pathways. The cochlear mechanics has first principle descriptions, and so partial differential equations (PDEs) become a natural mathematical framework to initiate computation. However, in vivo cochlear dynamics is not a pure mechanical problem, and neural couplings are present to modify responses. To incorporate both aspects, a first-principle based PDE model was studied in 21] for voice signal processing, where the neural aspect is introduced in the model phenomenologically. The first principle based PDE approach is more systematic compared with filter bank method [13], and has shown encouraging results. In [21], time domain computation on multi-tone inputs revealed tonal suppressions in qualitative agreement with earlier neural experimental findings.

In this paper, we shall analyze the well-posedness and construct multitone solutions of such PDE models in the form:

$$
\begin{aligned}
& p_{x x}-N u_{t t}=\epsilon_{s}(x) u_{t}, \quad x \in(0, L), \\
& p=m u_{t t}+r\left(x,|u|,\left|u_{t}\right|\right) u_{t}+s(x) u
\end{aligned}
$$

where $p$ is the fluid pressure difference across the basilar membrane (BM), $u$ the BM displacement, $L$ the longitudinal length of BM; $N$ a constant depending on fluid density and cochlear channel size; $\epsilon_{s}(x) \geq 0$ is the damping of longitudinal fluid motion; $m, r, s$ are the mass, damping, and stiffness of BM per unit area, with $m$ a constant, $s$ a continuously differentiable nonnegative function of $x$. The coefficient $r$ is a nonlinear function(al) of $x, u, u_{t}$ :

$$
r\left(x,|u|,\left|u_{t}\right|\right)=r_{a}\left(\left|u_{t}\right|^{2}\right)+\gamma \int_{0}^{L} P\left(\left|u\left(x^{\prime}, t\right)\right|\right) K\left(x-x^{\prime}\right) d x^{\prime} .
$$

Here: $(\mathrm{H} 1) r_{a}(\cdot)$ is the local part of BM damping, it is a nonnegative continuously differentiable monotone increasing function, $r_{a}(0)>0$. In the nonlocal BM damping: $(\mathrm{H} 2) K=K(x)$ is a localized Lipschitz continuous kernel function with total integral over $x \in R^{1}$ equal to 1 ; (H3) $P(\cdot)$ is a nonnegative continuously differentiable function such that for some constant $C>0$ :

$$
P(0)=0, P(q) \leq C\left(1+q^{2}\right), \quad \forall q \geq 0 .
$$


The boundary and initial conditions of the system are:

$$
\begin{aligned}
& p_{x}(0, t)=T_{M} p_{T}(t) \equiv f(t), \quad p(L, t)=0, \\
& u(x, 0)=u_{0}(x), u_{t}(x, 0)=u_{1}(x),
\end{aligned}
$$

where the initial data is such that $\left(u_{0}, u_{1}\right)(L)=(0,0) ; p_{T}(t)$ is the input sound pressure at the eardrum; and $T_{M}$ is a bounded linear map modeling functions of middle ear, with output depending on the frequency content of $p_{T}(t)$. If $p_{T}=\sum_{j=1}^{J_{M}} A_{j} \exp \left\{i \omega_{j} t\right\}+$ c.c., a multi-tone input, c.c denoting complex conjugate, $J_{M}$ a positive integer, then $T_{M} p_{T}(t)=\sum_{j=1}^{J_{M}} B_{j} \exp \left\{i \omega_{j} t\right\}+c . c$, where $B_{j}=a_{M}\left(\omega_{j}\right) A_{j}$, c.c for complex conjugate, $a_{M}(\cdot)$ a scaling function built from the filtering characteristics of the middle ear [5].

Cochlear modeling has had a long history, and various linear models have been studied at length by analytical and numerical methods, [8], 10] and references therein. A brief derivation of the cochlear model of the transmission line type, e.g. the linear portion of (1.1)-(1.6), is nicely presented in [18] based on fluid and elasticity equations.

It has been realized that nonlinearity is essential for multitone interactions, 6, 9, 2, 4, etc. Nonlinearity could be introduced phenomenologically based on spreading of electrical and neural activities between hair cells at different BM locations suggested by experimental data, [7], [3]. Such a treatment turned out to be efficient for signal processing purpose [21], and (1.3)-(1.4) is a generalization of existing nonlinearities [7], [3], [19].

Multitone solutions require one to perform numerical computation in the time domain. The model system (1.1)-(1.6) is dispersive, and long waves tend to propagate with little decay from entrance point $x=0$ (stapes) to the exit $x=L$ (helicotrama). The function $\epsilon_{s}(x)$ is supported near $x=L$, its role in numerics is to suck out the long waves accumulating near the exit [21]. Selective positive or negative damping has been a novel way to filter images in PDE method of image processing [15]. In analysis of model solutions that concern mainly with interior properties however, we shall set $\epsilon_{s}$ to zero for technical convenience.

The rest of the paper is organized as follows. In section 2, we perform energy estimates of solutions for the model system (1.1)-(1.6), prove the global well-posedness and obtain growth and uniform bounds in Sobolev spaces. In section 3, we construct exact multi-frequency solutions when $\gamma$ is small enough and nonlinearity is cubic, using contraction mapping in a suitable Banach space. The constructed solutions contain all linear integral combinations of input frequencies. In section 4 , for two input tones with frequencies $f_{1}$ and $f_{2}\left(f_{1}<f_{2}\right)$, we illustrate numerically the generated combination tones $2 f_{1}-f_{2}$ and $2 f_{2}-f_{1}$ on power spectral density plots at selected points on BM. These tones are heard on musical instruments (piano and violin), in particular, $2 f_{1}-f_{2}$ is known as the Tartini tone. The conclusions are in section 5 . 


\section{Global Well-Posedness and Estimates}

Let us consider the initial boundary value problem (IBVP) formed by (1.1)-(1.6) and show that solutions exist uniquely in a proper function space for all time. To this end, it is convenient to work with the equivalent integral form of the equations. It follows from (1.1) and (1.5) that:

$$
\begin{gathered}
p_{x}=\int_{0}^{x}\left(N u_{t t}+\epsilon_{s}(x) u_{t}\right) d x+f(t) \\
-p(t, x)=\int_{x}^{L} d x^{\prime} \int_{0}^{x^{\prime}}\left(N u_{t t}+\epsilon_{s} u_{t}\right) d x^{\prime \prime}+f(t)(L-x) .
\end{gathered}
$$

Combining (1.2) and (2.1), we get:

$$
m u_{t t}+\int_{x}^{L} d x^{\prime} \int_{0}^{x^{\prime}}\left(N u_{t t}+\epsilon_{s} u_{t}\right) d x^{\prime \prime}+f(t)(L-x)=-r\left(x,|u|,\left|u_{t}\right|\right) u_{t}-s(x) u
$$

with initial data (1.6). Let $w=\left(w_{1}, w_{2}\right)=\left(u, u_{t}\right)$, and write (2.2) into the system form:

$$
\begin{aligned}
w_{1, t} & =w_{2} \\
m w_{2, t}+\int_{x}^{L} d x^{\prime} \int_{0}^{x^{\prime}} N w_{2, t} d x^{\prime \prime}= & -\int_{x}^{L} d x^{\prime} \int_{0}^{x^{\prime}} \epsilon_{s} w_{2} d x^{\prime \prime} \\
& -r\left(x,\left|w_{1}\right|,\left|w_{2}\right|\right) w_{2}-s(x) w_{1}+f(t)(x-L) .
\end{aligned}
$$

The related integral form is:

$$
\begin{aligned}
w_{1}= & u_{0}+\int_{0}^{t} w_{2}(x, \tau) d \tau \\
A w_{2}= & A u_{1}-\int_{0}^{t} d \tau \int_{x}^{L} d x^{\prime} \int_{0}^{x^{\prime}} \epsilon_{s} w_{2} d x^{\prime \prime} \\
& -\int_{0}^{t} d \tau r\left(x,\left|w_{1}\right|,\left|w_{2}\right|\right) w_{2}-s(x) \int_{0}^{t} w_{1} d \tau+(x-L) \int_{0}^{t} f(\tau) d \tau
\end{aligned}
$$

where $A: L^{2}([0, L]) \rightarrow L^{2}([0, L])$ is a bounded self-adjoint linear operator:

$$
A g \equiv m g+\int_{x}^{L} d x^{\prime} \int_{0}^{x^{\prime}} N g d x^{\prime \prime} \equiv m g+\tilde{A} g
$$

To see the self-adjointness of $A$, let $g, h \in L^{2}([0, L])$, then $(\tilde{A} g, h)=(g, \tilde{A} h)$ or:

$$
\begin{aligned}
& \int_{0}^{L}\left(\int_{x}^{L} d x^{\prime} \int_{0}^{x^{\prime}} g\left(x^{\prime \prime}\right) d x^{\prime \prime}\right) h(x) d x=\int_{0}^{L}\left(\int_{x}^{L} d x^{\prime} \int_{0}^{x^{\prime}} g\left(x^{\prime \prime}\right) d x^{\prime \prime}\right) d \int_{0}^{x} h \\
= & \int_{0}^{L}\left(\int_{0}^{x} d x^{\prime} g\left(x^{\prime}\right)\right)\left(\int_{0}^{x} d x^{\prime} h\left(x^{\prime}\right)\right) d x,
\end{aligned}
$$


hence $(A g, h)_{L^{2}}=(g, A h)_{L^{2}}$. Clearly, $A$ is bounded; also $(A \cdot, \cdot)=(A \cdot, \cdot)_{L^{2}}$ is an equivalent square $L^{2}$ norm:

$$
\begin{aligned}
& (A g, g)=m\|g\|_{2}^{2}+N \int_{0}^{L}\left(\int_{0}^{x} g\right)^{2} d x \geq m\|g\|_{2}^{2} \\
& (A g, g) \leq m\|g\|_{2}^{2}+N L^{2}\|g\|_{2}^{2}=\left(m+N L^{2}\right)\|g\|_{2}^{2}
\end{aligned}
$$

Moreover, $A$ has a bounded inverse. To see this, note that $A-m I d$ is a compact operator on $L^{2}([0, L])$, so Riesz-Schauder theory [22] says that the spectrum of $A$ can have only eigenvalues of finite multiplicities except at number $m$. On the other hand, zero cannot be an eigenvalue of $A$, as $(A g, g) \geq m\|g\|_{2}^{2}$. The bounded inverse of $A$ follows, and we denote it by $A^{-1}$ below.

Now we establish the global existence of solutions of (2.3)-(2.4) in the function space $C\left([0, \infty) ;\left(H^{1}([0, L])\right)^{2}\right)$. It is straightforward to show by contraction mapping principle that if $\left\|\left(u_{0}, u_{1}\right)\right\|_{H^{1}}<\infty$, there is a time $t_{*}$ such that (2.5) has a unique solution in $C\left(\left[0, t_{*}\right) ;\left(H^{1}([0, L])\right)^{2}\right)$ under our assumptions on the nonlinearities. Such a solution in fact lies in $C^{1}\left(\left[0, t_{*}\right) ;\left(H^{1}([0, L])\right)^{2}\right)$, and obeys the differential form of equations (2.3) $-(2.4)$, with both sides interpreted in the $H^{1}$ sense. Taking the limit $x \rightarrow L$, we find that the system (2.3)-(2.4) reduces to the ODE system:

$$
\begin{aligned}
& w_{1, t}=w_{2}, \\
& w_{2, t}=-r(t) w_{2}-s(L) w_{1},
\end{aligned}
$$

with initial data $\left(w_{1}, w_{2}\right)(L, 0)=(0,0)$, hence $\left(w_{1}, w_{2}\right)(L, t)=(0,0), \forall t \in\left(0, t_{*}\right)$.

Let us derive global in time estimates of solutions in $H^{1}$ to extend the local solutions to global ones $\left(\right.$ so $\left.t_{*}=\infty\right)$. The left hand side of (2.4) is just $\left(A w_{2}\right)_{t}$, and:

$$
\left(w_{2},\left(A w_{2}\right)_{t}\right)=\left(w_{2}, A\left(w_{2}\right)_{t}\right)=\left(A w_{2}, w_{2, t}\right)=\left(w_{2, t}, A w_{2}\right)
$$

SO:

$$
\frac{d}{d t}\left(A w_{2}, w_{2}\right)=\left(w_{2, t}, A w_{2}\right)+\left(A w_{2, t}, w_{2}\right)=2\left(w_{2}, A w_{2, t}\right)
$$

hence $\frac{1}{2} \frac{d}{d t}\left(A w_{2}, w_{2}\right)=\left(w_{2}, A w_{2, t}\right)$. Multiplying (2.3) by $w_{1}$, (2.4) by $w_{2}$, adding the two expressions and integrating over $[0, L]$, we estimate with Cauchy-Schwarz inequality:

$$
\begin{aligned}
& \left(w_{1}, w_{1, t}\right)+\left(w_{2},\left(A w_{2}\right)_{t}\right)=\frac{1}{2} \frac{d}{d t}\left(\left(w_{1}, w_{1}\right)+\left(A w_{2}, w_{2}\right)\right) \\
= & -\left(\int_{x}^{L} d x^{\prime} \int_{0}^{x^{\prime}} \epsilon_{s} w_{2} d x^{\prime \prime}, w_{2}\right)-\left(r w_{2}, w_{2}\right) \\
& +\left(w_{2}, w_{1}\right)-\left(s w_{1}, w_{2}\right)+\left(f(t)(x-L), w_{2}\right) \\
\leq & -r_{a}(0)\left\|w_{2}\right\|_{2}^{2}+r_{a}(0)\left\|w_{2}\right\|_{2}^{2}+\frac{1}{4 r_{a}(0)}\|f(t)(x-L)\|_{2}^{2}
\end{aligned}
$$




$$
\begin{aligned}
& -\int_{0}^{L}\left(\int_{0}^{x} \epsilon_{s} w_{2}\right)\left(\int_{0}^{x} w_{2}\right) d x+\|(1-s)\|_{\infty}\left\|w_{2}\right\|_{2}\left\|w_{1}\right\|_{2} \\
\leq & \frac{1}{12 r_{a}(0)}|f(t)|^{2} L^{3}+L^{2}\left\|\epsilon_{s}\right\|_{2}\|w\|_{2}^{2}+\frac{1}{2}\|1-s\|_{\infty}\left(\left\|w_{1}\right\|_{2}^{2}+\left\|w_{2}\right\|_{2}^{2}\right) \\
\leq & \left(\frac{1}{2}\|1-s\|_{\infty}+L^{2}\left\|\epsilon_{s}\right\|_{2}\right)\left\|w_{2}\right\|_{2}^{2}+\frac{1}{2}\|1-s\|_{\infty}\left\|w_{1}\right\|_{2}^{2}+\frac{1}{12 r_{a}(0)}|f(t)|^{2} L^{3} .
\end{aligned}
$$

Let $C_{1}=\max \left(\frac{1}{m}\|1-s\|_{\infty}+\frac{2 L^{2}}{m}\left\|\epsilon_{s}\right\|_{2}, \frac{1}{2}\|1-s\|_{\infty}\right)$, and $2 E=\left(w_{1}, w_{1}\right)+\left(A w_{2}, w_{2}\right)$, we have from (2.9):

$$
\frac{d E}{d t} \leq C_{1} E+\frac{1}{12 r_{a}(0)}|f(t)|^{2} L^{3}
$$

or:

$$
E(t) \leq E(0)+C_{1} \int_{0}^{t} E(s) d s+\frac{L^{3}}{12 r_{a}(0)} \int_{0}^{t}|f|^{2}\left(s^{\prime}\right) d s^{\prime} .
$$

Gronwall inequality implies:

$$
E(t) \leq\left(E(0)+\frac{L^{3}}{12 r_{a}(0)} \int_{0}^{t}|f|^{2}\left(t^{\prime}\right) d t^{\prime}\right) e^{C_{1} t}
$$

or:

$$
\left\|\left(w_{1}, w_{2}\right)\right\|_{2}^{2} \leq \min (1, m)^{-1}\left(E(0)+\frac{L^{3}}{12 r_{a}(0)} \int_{0}^{t}|f|^{2}\left(t^{\prime}\right) d t^{\prime}\right) e^{C_{1} t} .
$$

Next we obtain the gradient estimates. Differentiating (2.3) $-(2.4)$ in $x$ gives:

$$
\begin{aligned}
& \frac{d}{d t} w_{1, x}=w_{2, x} \\
& \frac{d}{d t}\left(m w_{2, x}-N \int_{0}^{x} w_{2}\left(x^{\prime}, \cdot\right) d x^{\prime}\right)=\int_{0}^{x} \epsilon_{s}\left(x^{\prime}\right) w_{2}\left(x^{\prime}\right) d x^{\prime}-r w_{2, x}-s^{\prime} w_{1}-s w_{1, x}+f(t) \\
& -\left(2 r_{a}^{\prime} w_{2} w_{2, x}+\int_{0}^{L} P\left(w_{1}\right)\left(x^{\prime}, t\right) K_{x}\left(x-x^{\prime}\right) d x^{\prime}\right) w_{2} .
\end{aligned}
$$

Multiplying (2.12) and (2.13) by $w_{1, x}$ and $w_{2, x}$, and integrating over $x \in[0, L]$, we find:

$$
\begin{aligned}
& \frac{1}{2} \frac{d}{d t}\left(\left\|w_{1, x}\right\|_{2}^{2}+m\left\|w_{2, x}\right\|_{2}^{2}\right)=N \frac{d}{d t} \int_{0}^{L} w_{2, x} \int_{0}^{x} w_{2}\left(x^{\prime}, \cdot\right) d x^{\prime}+\left(w_{1, x}, w_{2, x}\right) \\
& +\left(w_{2, x}, \int_{0}^{x} \epsilon_{s} w_{2}\right)-\int_{0}^{L} r w_{2, x}^{2}-\int_{0}^{L}\left(2 r_{a}^{\prime} w_{2} w_{2, x}+\int_{0}^{L} P\left(w_{1}\right) K_{x}\right) w_{2} w_{2, x} \\
& -\int_{0}^{L} s^{\prime} w_{1} w_{2, x}-\int_{0}^{L} s w_{1, x} w_{2, x}+f(t) \int_{0}^{L} w_{2, x} .
\end{aligned}
$$

The integral in the first term of the right hand side of (2.14) equals:

$$
\frac{N}{2} \int_{0}^{L} d x w_{2, x}(x, \cdot) \int_{0}^{x} w_{2}\left(x^{\prime}, \cdot\right) d x^{\prime}=-\frac{N}{2} \int_{0}^{L} w_{2}^{2}\left(x^{\prime}, t\right) d x^{\prime},
$$


where we applied integration by parts once and $w_{2}(L, t)=0$.

The other terms are estimated as follows:

$$
\begin{aligned}
& -\int_{0}^{L} d x\left(\int_{0}^{L} P\left(w_{1}\right)\left(x^{\prime}, \cdot\right) K_{x}\left(x-x^{\prime}\right) d x^{\prime}\right) w_{2} w_{2, x} \\
\leq & C \int_{0}^{L}\left|w_{2} w_{2, x}\right| \int_{0}^{L}\left(1+\left|w_{1}\right|^{2}\right)\left|K_{x}\right|\left(x-x^{\prime}\right) d x^{\prime} \\
\leq & C\left(1+\left\|w_{1}\right\|_{2}^{2}\right) \int_{0}^{L}\left|w_{2} w_{2, x}\right| \\
\leq & C\left(1+\left\|w_{1}\right\|_{2}^{2}\right)\left\|w_{2}\right\|_{2}\left\|w_{2, x}\right\|_{2} \\
\leq & \delta_{0}\left\|w_{2, x}\right\|_{2}^{2}+\frac{1}{4 \delta_{0}} C^{2}\left(1+\left\|w_{1}\right\|_{2}^{2}\right)^{2}\left\|w_{2}\right\|_{2}^{2},
\end{aligned}
$$

for any $\delta_{0}>0, C=C\left(\left\|K_{x}\right\|_{\infty}\right)$, by (1.4). Integration by parts and $w_{2}(L, t)=0$ give:

$$
\left(w_{2, x}, \int_{0}^{x} \epsilon_{s} w_{2}\right)=-\int_{0}^{L} \epsilon_{s} w_{2}^{2} d x \leq 0
$$

Estimate with Cauchy-Schwarz inequalities to get:

$$
\begin{aligned}
& -\int_{0}^{L} r w_{2, x}^{2}-2 \int_{0}^{L} r_{a}^{\prime} w_{2}^{2} w_{2, x}^{2} \leq-r_{a}(0)\left\|w_{2, x}\right\|_{2}^{2} ; \\
& -\int_{0}^{L} s^{\prime} w_{1} w_{2, x} d x-\int_{0}^{L} s w_{1, x} w_{2, x}+\left(w_{1, x}, w_{2, x}\right) \\
\leq & \|1-s\|_{\infty}\left\|w_{1, x}\right\|_{2}\left\|w_{2, x}\right\|_{2}+\left\|s^{\prime}\right\|_{\infty}\left\|w_{1}\right\|_{2}\left\|w_{2, x}\right\|_{2} \\
\leq & 2 \delta_{0}\left\|w_{2, x}\right\|_{2}^{2}+\frac{1}{16 \delta_{0}}\|1-s\|_{\infty}^{2}\left\|w_{1, x}\right\|_{2}^{2}+\frac{1}{16 \delta_{0}}\left\|s^{\prime}\right\|_{\infty}^{2}\left\|w_{1}\right\|_{2}^{2} ; \\
& f(t) \int_{0}^{L} w_{2, x} \leq|f(t)| L^{1 / 2}\left\|w_{2, x}\right\|_{2} \leq \delta_{0}\left\|w_{2, x}\right\|_{2}^{2}+\frac{1}{4 \delta_{0}}|f(t)|^{2} L .
\end{aligned}
$$

Combining (2.14)-(2.20), with $4 \delta_{0}=r_{a}(0) / 2$, we get:

$$
\frac{d}{d t} \frac{1}{2}\left(\left\|w_{1, x}\right\|_{2}^{2}+m\left\|w_{2, x}\right\|_{2}^{2}\right) \leq-\frac{N}{2} \frac{d}{d t}\left\|w_{2}\right\|_{2}^{2}-\frac{r_{a}(0)}{2}\left\|w_{2, x}\right\|_{2}^{2}+C_{2}(t)+C_{3}(t)\left\|w_{1, x}\right\|_{2}^{2},
$$

where:

$$
\begin{aligned}
C_{2}(t) & =\frac{|f(t)|^{2} L}{4 \delta_{0}}+\frac{\left\|s^{\prime}\right\|_{\infty}^{2}}{16 \delta_{0}}\left\|w_{1}\right\|_{2}^{2}+\frac{C^{2}}{4 \delta_{0}}\left(1+\left\|w_{1}\right\|_{2}^{2}\right)^{2}\left\|w_{2}\right\|_{2}^{2}, \\
C_{3}(t) & =\frac{1}{16 \delta_{0}}\|1-s\|_{\infty}^{2}
\end{aligned}
$$

and $\|\left(w_{1}, w_{2} \|_{2}\right.$ are bounded as in (2.11). Integrating (2.21) over $t \in[0, T]$, we find:

$$
m^{\prime}\left\|\left(w_{1, x}, w_{2, x}\right)\right\|_{2}^{2}(T)+\frac{N}{2}\left\|w_{2}\right\|_{2}^{2} \leq C_{4}+\int_{0}^{T} C_{2}\left(t^{\prime}\right) d t^{\prime}+\int_{0}^{T} C_{3}\left(t^{\prime}\right)\left\|\left(w_{1}, w_{2}\right)_{x}\right\|_{2}^{2},
$$


where $m^{\prime} \equiv \frac{1}{2} \min (1, m)$,

$$
C_{4}=\frac{1}{2} \max (1, m)\left\|\left(u_{0, x}, u_{1, x}\right)\right\|_{2}^{2}+\frac{N}{2}\left\|u_{1}\right\|_{2}^{2} ;
$$

and Gronwall inequality implies:

$$
\left\|\left(w_{1, x}, w_{2, x}\right)\right\|_{2}^{2}(T) \leq \frac{1}{m^{\prime}}\left(C_{4}+\int_{0}^{T} C_{2}(t) d t\right) \exp \left\{\frac{1}{m^{\prime}} \int_{0}^{T} C_{3}(t) d t\right\} .
$$

We see from (2.4) that $w_{2, t} \in C\left([0, \infty) ; H^{1}([0, L])\right)$, hence pressure $p \in C\left([0, \infty) ; H^{3}([0, L])\right)$ from (2.1). We have thus shown:

Theorem 2.1 Under the growth condition (1.4) and the initial boundary conditions (1.5) and (1.6), the model cochlear system (1.1)-(1.3) has unique global solutions:

$$
\left(u, u_{t}, p\right) \in C\left([0, \infty) ;\left(H^{1}([0, L])\right)^{2} \times H^{3}([0, L])\right) .
$$

The estimates can be improved with the additional assumption:

$$
s(x) \geq s_{0}>0, \quad \forall x \in[0, L] ; \quad\left\|\epsilon_{s}\right\|_{2}<\frac{3 r_{a}(0)}{2 L^{\frac{3}{2}}} .
$$

Theorem 2.2 (Growth Bounds) Under the additional assumption (2.27), the global solutions in Theorem 2.1 satisfy the bounds:

$$
\begin{aligned}
\left\|\left(u, u_{t}\right)\right\|_{2}^{2}+\int_{0}^{t}\left\|u_{t}\right\|_{2}^{2}\left(t^{\prime}\right) d t^{\prime} & \leq a_{1}+a_{2} \int_{0}^{t}\left|f\left(t^{\prime}\right)\right|^{2} d t^{\prime}, \\
\left\|\left(u_{x}, u_{x, t}\right)\right\|_{2}^{2}+\int_{0}^{t}\left\|u_{x, t}\right\|_{2}^{2}\left(t^{\prime}\right) d t^{\prime} & \leq a_{3}+a_{4} \int_{0}^{t}\left(1+\int_{0}^{t^{\prime}}\left|f\left(t^{\prime \prime}\right)\right|^{2} d t^{\prime \prime}\right)^{3} d t^{\prime},
\end{aligned}
$$

for some positive constants $a_{i}, i=1,2,3,4$.

Proof: Multiplying (2.3) by $s(x) w_{1}$, and (2.4) by $w_{2}$, adding the two expressions and integrating over $[0, L]$, we estimate with Cauchy-Schwarz inequality:

$$
\begin{aligned}
& \left(s w_{1}, w_{1, t}\right)+\left(w_{2},\left(A w_{2}\right)_{t}\right)=-\left(\int_{x}^{L} d x^{\prime} \int_{0}^{x^{\prime}} \epsilon_{s} w_{2} d x^{\prime \prime}, w_{2}\right) \\
& -\left(r w_{2}, w_{2}\right)+\left(f(t)(x-L), w_{2}\right) \\
\leq & -\left(\int_{0}^{x} w_{2}\left(x^{\prime}\right) d x^{\prime}, \int_{0}^{x} \epsilon_{s}\left(x^{\prime}\right) w_{2}\left(x^{\prime}\right) d x^{\prime}\right)-r_{a}(0)\left\|w_{2}\right\|_{2}^{2}+f(t)\left(w_{2},(x-L)\right) \\
\leq & -\left(r_{a}(0)-\frac{2}{3} L^{3 / 2}\left\|\epsilon_{s}\right\|_{2}\right)\left\|w_{2}\right\|_{2}^{2}+\delta\left\|w_{2}\right\|_{2}^{2}+\frac{|f|^{2}}{4 \delta}\|(x-L)\|_{2}^{2} .
\end{aligned}
$$


Choose $2 \delta=r_{a}(0)-\frac{2}{3} L^{3 / 2}\left\|\epsilon_{s}\right\|_{2}>0$ to find:

$$
\frac{1}{2} \frac{d}{d t}\left(\left(s w_{1}, w_{1}\right)+\left(A w_{2}, w_{2}\right)\right) \leq-\delta\left\|w_{2}\right\|_{2}^{2}+\frac{|f|^{2}}{4 \delta} \frac{L^{3}}{3} .
$$

So:

$$
\frac{1}{2}\left(\left(s w_{1}, w_{1}\right)+\left(A w_{2}, w_{2}\right)\right)(t) \leq-\delta \int_{0}^{t}\left\|w_{2}\right\|_{2}^{2}+c_{0}+c_{1} \int_{0}^{t}\left|f\left(t^{\prime}\right)\right|^{2} d t^{\prime} .
$$

where $c_{0}=\frac{1}{2}\left(\left(s w_{1}, w_{1}\right)+\left(A w_{2}, w_{2}\right)\right)(0)$, and $c_{1}=\frac{L^{3}}{12 \delta}$. Hence,

$$
\begin{aligned}
\frac{\min \left(s_{0}, m\right)}{2}\left\|\left(w_{1}, w_{2}\right)\right\|_{2}^{2} & \leq c_{0}+c_{1} \int_{0}^{t}\left|f\left(t^{\prime}\right)\right|^{2} d t^{\prime} \\
\int_{0}^{t}\left\|w_{2}\right\|_{2}^{2}\left(t^{\prime}\right) d t^{\prime} & \leq \delta^{-1} c_{0}+\delta^{-1} \int_{0}^{t}\left|f\left(t^{\prime}\right)\right|^{2} d t^{\prime} .
\end{aligned}
$$

In particular, if $f(t)$ a bounded continuous function, (2.32) gives the growth bounds:

$$
\left\|\left(w_{1}, w_{2}\right)\right\|_{2} \leq O\left(t^{1 / 2}\right), \int_{0}^{t}\left\|w_{2}\right\|_{2}^{2}\left(t^{\prime}\right) d t^{\prime} \leq O(t)
$$

implying that $\left\|w_{2}\right\|_{2}$ has a bounded time averaged $L^{2}$ norm square.

Similarly, we improve the gradient estimates. Multiplying (2.12) by $s w_{1, x},(2.13)$ by $w_{2, x}$, integrating over $x \in[0, L]$, we cancel out the two integrals on $s(x) w_{1, x} w_{2, x}$. Proceeding as before, we arrive at:

$$
\frac{d}{d t} \frac{1}{2}\left(\left(s w_{1, x}, w_{1, x}\right)+m\left\|w_{2, x}\right\|_{2}^{2}\right) \leq-\frac{N}{2} \frac{d}{d t}\left\|w_{2}\right\|_{2}^{2}-\frac{r_{a}(0)}{2}\left\|w_{2, x}\right\|_{2}^{2}+C_{2}(t),
$$

and so integrating over $[0, T]$ gives:

$$
\begin{aligned}
& \frac{1}{2}\left(\left(s w_{1, x}, w_{1, x}\right)+m\left\|w_{2, x}\right\|_{2}^{2}\right)(T)+\frac{r_{a}(0)}{2} \int_{0}^{T}\left\|w_{2, x}\right\|_{2}^{2}\left(t^{\prime}\right) d t^{\prime}+\frac{N}{2}\left\|w_{2}\right\|_{2}^{2}(T) \\
\leq & \frac{1}{2}\left(\left(s u_{0, x}, u_{0, x}\right)+m\left\|u_{1, x}\right\|_{2}^{2}\right)+\frac{N}{2}\left\|u_{1}\right\|_{2}^{2}+\int_{0}^{T} C_{2}\left(t^{\prime}\right) d t^{\prime} \equiv C_{5,0}+\int_{0}^{T} C_{2}\left(t^{\prime}\right) d t^{\prime} .
\end{aligned}
$$

If $s(x) \geq s_{0}>0$, then:

$$
\left\|\left(w_{1, x}, w_{2, x}\right)\right\|_{2}^{2}(T)+\frac{r_{a}(0)}{2 m^{\prime \prime}} \int_{0}^{T}\left\|w_{2, x}\right\|_{2}^{2}(t) d t \leq \frac{1}{m^{\prime \prime}}\left(C_{5,0}+\int_{0}^{T} C_{2}(t) d t\right),
$$

where $m^{\prime \prime}=\frac{1}{2} \min \left(s_{0}, m\right)$. Substituting (2.32) in (2.36) gives (2.28). In particular, for a bounded continuous $f(t)$,

$$
\left\|\left(w_{1, x}, w_{2, x}\right)\right\|_{2}^{2}(T)+\frac{1}{2 m^{\prime \prime}} \int_{0}^{T}\left\|w_{2, x}\right\|_{2}^{2}(t) d t \leq O\left(T^{3}\right)
$$


The proof is finished.

If the nonlinear damping functions are bounded, i.e:

$$
r_{a}(\xi) \leq C_{6}, r_{a}^{\prime}(\xi) \xi \leq C_{6}, P(\xi) \leq C_{6}, \forall \xi \geq 0
$$

for some positive constant $C_{6}$, then we have:

Theorem 2.3 (Uniform Bounds) Under the assumptions 2.27) and (2.38), and that $f(t)$ is a bounded continuous function, the global solutions in Theorem 2.1 are uniformly bounded:

$$
\left\|\left(u, u_{t}\right)\right\|_{H^{1}}+\|p\|_{H^{3}} \leq C_{7}<\infty, \quad \forall t \geq 0
$$

for some positive constant $C_{1}$. Moreover, the dynamics admit an absorbing ball:

$$
\limsup _{t \rightarrow \infty}\left(\left\|\left(u, u_{t}\right)\right\|_{H^{1}}+\|p\|_{H^{3}}\right) \leq C_{8}
$$

where $C_{8}$ is independent of initial data.

See [19] for an example of a bounded damping function. The energy inequality (2.30) lacks a term like - const. $\left\|w_{1}\right\|_{2}^{2}$ on the right hand side, and so is insufficient to provide uniform bounds. The idea is to bring out the skew symmetric part of the system.

Proof: multiply (2.3) by $m w_{2}$, (2.4) by $w_{1}$, integrate over $x \in[0, L]$, and add the resulting expressions to get:

$$
\begin{aligned}
m\left(w_{1}, w_{2}\right)_{t}+\left(\tilde{A} w_{2, t}, w_{1}\right) & =m\left\|w_{2}\right\|_{2}^{2}-\left(s w_{1}, w_{1}\right)-\left(r w_{2}, w_{1}\right) \\
& +\left(f(t)(x-L), w_{1}\right)-\left(w_{1}, \int_{x}^{L} d x^{\prime} \int_{0}^{x^{\prime}} \epsilon_{s} w_{2} d x^{\prime \prime}\right) .
\end{aligned}
$$

Using the identity:

$$
\frac{d}{d t}\left(\tilde{A} w_{2}, w_{1}\right)=\left(\tilde{A} w_{2, t}, w_{1}\right)+\left(\tilde{A} w_{2}, w_{1, t}\right)=\left(\tilde{A} w_{2, t}, w_{1}\right)+\left(\tilde{A} w_{2}, w_{2}\right)
$$

we have:

$$
\begin{aligned}
\frac{d}{d t}\left[m\left(w_{1}, w_{2}\right)+\left(\tilde{A} w_{2}, w_{1}\right)\right] & =\left(\tilde{A} w_{2}, w_{2}\right)+m\left\|w_{2}\right\|_{2}^{2}-\left(s w_{1}, w_{1}\right)-\left(r w_{2}, w_{1}\right) \\
& +\left(f(t)(x-L), w_{1}\right)-\left(w_{1}, \int_{x}^{L} d x^{\prime} \int_{0}^{x^{\prime}} \epsilon_{s} w_{2} d x^{\prime \prime}\right) \\
\leq & \left(N L^{2}+m\right)\left\|w_{2}\right\|_{2}^{2}-s_{0}\left\|w_{1}\right\|_{2}^{2}+C_{6}\left\|w_{2}\right\|_{2}\left\|w_{1}\right\|_{2} \\
& +|f| L^{3 / 2}\left\|w_{1}\right\|_{2}+L^{3 / 2}\left\|\epsilon_{2}\right\|_{2}\left\|w_{1}\right\|_{2}\left\|w_{2}\right\|_{2} .
\end{aligned}
$$


Apply Cauchy-Schwarz to polarize the last three terms to get:

$$
\begin{aligned}
C_{6}\left\|w_{2}\right\|_{2}\left\|w_{1}\right\|_{2} & \leq \frac{C_{6}^{2}}{s_{0}}\left\|w_{2}\right\|_{2}^{2}+\frac{s_{0}}{4}\left\|w_{1}\right\|_{2}^{2}, \\
|f| L^{3 / 2}\left\|w_{1}\right\|_{2} & \leq|f|^{2} L^{3} / s_{0}+\frac{s_{0}}{4}\left\|w_{1}\right\|_{2}^{2}, \\
L^{3 / 2}\left\|\epsilon_{2}\right\|_{2}\left\|w_{1}\right\|_{2}\left\|w_{2}\right\|_{2} & \leq \frac{L^{3}\left\|\epsilon_{2}\right\|_{2}^{2}}{s_{0}}\left\|w_{2}\right\|_{2}^{2}+\frac{s_{0}}{4}\left\|w_{1}\right\|_{2}^{2},
\end{aligned}
$$

it follows from (2.40) that:

$$
\frac{d}{d t}\left[m\left(w_{1}, w_{2}\right)+\left(\tilde{A} w_{2}, w_{1}\right)\right] \leq C_{9}\left\|w_{2}\right\|_{2}^{2}-\frac{s_{0}}{4}\left\|w_{1}\right\|_{2}^{2}+|f|^{2} L^{3} / s_{0},
$$

where:

$$
C_{9}=N L^{2}+m+\frac{C_{6}^{2}}{s_{0}}+\frac{L^{3}\left\|\epsilon_{2}\right\|_{2}^{2}}{s_{0}} .
$$

Multiplying (2.30) by a positive constant $C_{p}$ and adding the resulting inequality to (2.42), we find:

$$
\frac{d}{d t} E_{p} \leq\left(-\delta C_{p}+C_{9}\right)\left\|w_{2}\right\|_{2}^{2}-\frac{s_{0}}{4}\left\|w_{1}\right\|_{2}^{2}+|f|^{2}\left(\frac{L^{3}}{s_{0}}+\frac{C_{p} L^{3}}{12 \delta}\right)
$$

where:

$$
E_{p}=\frac{C_{p}}{2}\left(\left(s w_{1}, w_{1}\right)+\left(A w_{2}, w_{2}\right)\right)+m\left(w_{1}, w_{2}\right)+\left(\tilde{A} w_{2}, w_{1}\right) .
$$

Choose $C_{p}$ large enough so that $C_{p}>C_{9} / \delta$, and:

$$
\begin{aligned}
E_{p} & \geq \frac{C_{p}}{4}\left(\left(s w_{1}, w_{1}\right)+\left(A w_{2}, w_{2}\right)\right) \\
& \geq \min \left(s_{0}, m\right) \frac{C_{p}}{4}\left\|\left(w_{1}, w_{2}\right)\right\|_{2}^{2}
\end{aligned}
$$

SO:

$$
E_{p} \leq \frac{3 C_{p}}{4}\left(\left(s w_{1}, w_{1}\right)+\left(A w_{2}, w_{2}\right)\right) \leq \frac{3 C_{p}}{4} \max \left(\|s\|_{\infty}, m+N L^{2}\right)\left\|\left(w_{1}, w_{2}\right)\right\|_{2}^{2},
$$

thanks to (2.7), then for some positive constant $C_{10}$ :

$$
\frac{d}{d t} E_{p} \leq-C_{10} E_{p}+|f|^{2}\left(\frac{L^{3}}{s_{0}}+\frac{C_{p} L^{3}}{12 \delta}\right)
$$

The uniform bound on $\left\|\left(u, u_{t}\right)\right\|_{2}$ follows from (2.45). Moreover, the fact that $C_{10}$ is independent of initial data implies the absorbing ball property of $\left(u, u_{t}\right)$ in $L^{2}$ (i.e. the limsup as $t \rightarrow \infty$ is bounded independent of initial data). Equation (2.4) and $L^{2}$ invertibility of the operator $A$ imply a similar uniform bound on $u_{t t}$. Equation (2.1) in turn shows that $\|p\|_{H^{2}}$ is uniformly bounded and has the absorbing ball property as well. 
Now we proceed with gradient estimate of $\left(w_{1}, w_{2}\right)$. The symmetric inequality is just (2.34) but with $C_{2}$ uniform in time now. The skew symmetric inequality is obtained by multiplying $m w_{2, x}$ to (2.12) plus $w_{1, x}$ times (2.13), integrating over $x \in[0, L]$ :

$$
\begin{aligned}
& \frac{d}{d t}\left[m\left(w_{1, x}, w_{2, x}\right)\right]-N\left(w_{1, x}, \frac{d}{d t} \int_{0}^{x} w_{2}\left(x^{\prime}, t\right) d x^{\prime}\right) \\
& \leq\left(w_{1, x}, \int_{0}^{x} \epsilon_{s} w_{2}\right)-\left(r w_{2, x}, w_{1, x}\right)-\left(s^{\prime} w_{1}, w_{1, x}\right)-s_{0}\left\|w_{1, x}\right\|_{2}^{2} \\
& +f(t)\left(1, w_{1, x}\right)+2 C_{6}\left(\left|w_{1, x}\right|,\left|w_{2, x}\right|\right)+C_{11}\left(\left|w_{2}\right|,\left|w_{1, x}\right|\right) .
\end{aligned}
$$

The second term on the left hand side equals:

$$
N\left(w_{1}, w_{2, t}\right)=N \frac{d}{d t}\left(w_{1}, w_{2}\right)-N\left\|w_{2}\right\|_{2}^{2}
$$

It follows that:

$$
\frac{d}{d t}\left[m\left(w_{1, x}, w_{2, x}\right)+N\left(w_{1}, w_{2}\right)\right] \leq 4 C_{6}\left\|w_{1, x}\right\|_{2}\left\|w_{2, x}\right\|_{2}-\frac{s_{0}}{2}\left\|w_{1, x}\right\|_{2}^{2}+C_{12},
$$

for a positive constant $C_{12}$; or:

$$
\frac{d}{d t}\left[m\left(w_{1, x}, w_{2, x}\right)+N\left(w_{1}, w_{2}\right)\right] \leq \frac{16 C_{6}^{2}}{s_{0}}\left\|w_{2, x}\right\|_{2}^{2}-\frac{s_{0}}{4}\left\|w_{1, x}\right\|_{2}^{2}+C_{12}
$$

Multiplying a constant $C_{p}^{\prime}>0$ to (2.34) with $C_{2}$ constant, and adding the resulting inequality to (2.48), we get:

$$
\frac{d}{d t} E_{p}^{\prime} \leq-\frac{s_{0}}{4}\left\|w_{1, x}\right\|_{2}^{2}+C_{13}-\left(\frac{C_{p}^{\prime} r_{a}(0)}{2}-\frac{16 C_{6}^{2}}{s_{0}}\right)\left\|w_{2, x}\right\|_{2}^{2},
$$

where:

$$
E_{p}^{\prime}=\frac{C_{p}^{\prime}}{2}\left(\left(s w_{1, x}, w_{1, x}\right)+N\left\|w_{2}\right\|_{2}^{2}+m\left\|w_{2, x}\right\|_{2}^{2}\right)+m\left(w_{1, x}, w_{2, x}\right)+N\left(w_{1}, w_{2}\right),
$$

and $C_{13}=C_{2} C_{p}^{\prime}+C_{12}$. The term $\left(w_{1}, w_{2}\right)$ is bounded from above by constant times $\left\|\left(w_{1, x}, w_{2, x}\right)\right\|_{2}^{2}$ due to Poincaré inequality and so we can choose:

$$
C_{p}^{\prime}>\frac{32 C_{6}^{2}}{s_{0} r_{a}(0)}
$$

and large enough so that for some positive constants $C_{14}, C_{14}^{\prime}$ :

$$
C_{14}^{\prime}\left\|\left(w_{1, x}, w_{2, x}\right)\right\|_{2}^{2} \geq E_{p}^{\prime} \geq C_{14}\left\|\left(w_{1, x}, w_{2, x}\right)\right\|_{2}^{2} .
$$

Inequality (2.49) yields:

$$
\frac{d}{d t} E_{p}^{\prime} \leq-C_{15} E_{p}^{\prime}+C_{13}
$$

implying the uniform estimate on $\left\|\left(u_{x}, u_{t, x}\right)\right\|_{2}$ and the absorbing ball property. The uniform estimate and absorbing ball property on $\|p\|_{H^{3}}$ follows from (2.4) and (2.1). The proof is complete. 
Remark 2.1 The estimates in Theorem 2.3 imply that the evolution map denoted by $S(t)$ is relatively compact in the space $\left(u, u_{t}\right) \in\left(L^{2}([0, L])\right)^{2}$. Hence for any bounded initial data $\left(u, u_{t}\right)(0) \in\left(H^{1}([0, L])\right)^{2}$, the dynamics $\left(u, u_{t}\right)$ approach, in the space $\left(L^{2}([0, L])\right)^{2}$, the universal attractor $\mathbf{A}$ defined as:

$$
\mathbf{A}=\cap_{t>0} S(t) B_{\rho_{0}}
$$

where $B_{\rho_{0}}$ denotes the ball of radius $\rho_{0}$ in $\left(H^{1}([0, L])\right)^{2}$, the absorbing ball given by the estimates of Theorem 2.3.

\section{Multitone Solutions}

In this section, we consider special solutions to (1.1)-(1.2) that exhibit explicitly their frequency contents. For simplicity, let us assume that $\epsilon_{s}(x)=0$, and $s(x) \geq s_{0}>0, \forall x \in[0, L]$.

\subsection{Linear Waves}

First we consider the linear regime with $r=r_{0}$, a positive constant. Solutions are superpositions

of single frequency time harmonic waves of the form $p=P(x) e^{i \omega t}+$ c.c., $u=U(x) e^{i \omega t}+$ c.c, c.c complex conjugate, where $P$ and $U$ are complex functions that satisfy:

$$
\begin{array}{r}
P=\left(-m \omega^{2}+i r_{0} \omega+s(x)\right) U, \\
P_{x x}+N \omega^{2} U=0, \\
P_{x}(0)=P_{i n}, P(L)=0 .
\end{array}
$$

Let:

$$
(\alpha+i \beta)(x)=\frac{N \omega^{2}}{-m \omega^{2}+i r_{0} \omega+s(x)},
$$

so:

$$
(\alpha, \beta)=N \omega^{2}\left(-m \omega^{2}+s(x),-r_{0} \omega\right) /\left[\left(-m \omega^{2}+s(x)\right)^{2}+r_{0}^{2} \omega^{2}\right]
$$

then (3.1)-(3.3) is equivalent to:

$$
P_{x x}+(\alpha(x)+i \beta(x)) P=0
$$

subject to (3.3). If $\omega \neq 0, \beta \neq 0$. We show:

Lemma 3.1 The boundary value problem, (3.5) and (3.3), has a unique solution for all $\omega$ such that $\|P\|_{H^{2}([0, L])} \leq C\left|P_{\text {in }}\right|$, for some constant $C$ independent of $\omega$. 
Proof: Write $P=P_{i n}(x-L)+Q$, then $Q$ satisfies:

$$
Q_{x x}+(\alpha(x)+i \beta(x)) Q=-(\alpha(x)+i \beta(x))(x-L) P_{i n} \equiv\left(f_{1}+i f_{2}\right)(x),
$$

with boundary conditions $Q_{x}(0)=0, Q(L)=0$. The left hand side is a Fredholm operator on $Q$, so it is sufficient to prove that zero is not an eigenvalue, which follows from the estimate below. Write $Q=q_{1}+i q_{2}$, then:

$$
\left(\begin{array}{l}
q_{1} \\
q_{2}
\end{array}\right)_{x x}+\left(\begin{array}{rr}
\alpha & -\beta \\
\beta & \alpha
\end{array}\right)\left(\begin{array}{l}
q_{1} \\
q_{2}
\end{array}\right)=\left(\begin{array}{l}
f_{1} \\
f_{2}
\end{array}\right) .
$$

Multiplying (3.7) by $\left(q_{2},-q_{1}\right)$, we find:

$$
q_{1, x x} q_{2}-q_{1} q_{2, x x}-\beta q_{2}^{2}-\beta q_{1}^{2}=f_{1} q_{2}-f_{2} q_{1}
$$

which gives upon integrating over $x \in[0, L]$, integrating by parts:

$$
-\int_{0}^{L} \beta\left(q_{1}^{2}+q_{2}^{2}\right) d x=\int_{0}^{L}\left(f_{1} q_{2}-f_{2} q_{1}\right) d x .
$$

Multiplying (3.7) by $\left(q_{1}, q_{2}\right)$, integrating over $x \in[0, L]$, we have after integration by parts:

$$
-\left\|\left(q_{1, x}, q_{2, x}\right)\right\|_{2}^{2}+\int_{0}^{L} \alpha\left(q_{1}^{2}+q_{2}^{2}\right)=\int_{0}^{L}\left(f_{1} q_{1}+f_{2} q_{2}\right)
$$

It follows from (3.9) and Poincaré inequality that:

$$
\begin{aligned}
\left\|\left(q_{1}, q_{2}\right)\right\|_{2}^{2} & \leq L^{2}\left\|\left(q_{1, x}, q_{2, x}\right)\right\|_{2}^{2} \\
& \leq L^{2}\|\alpha\|_{\infty}\left\|\left(q_{1}, q_{2}\right)\right\|_{2}^{2}+L^{2}\left\|\left(f_{1}, f_{2}\right)\right\|_{2}\left\|\left(q_{1}, q_{2}\right)\right\|_{2}
\end{aligned}
$$

For $|\omega| \leq \omega_{0} \ll 1,\|\alpha\|_{\infty} \leq 2 N \omega^{2} / s_{0}, 2 \omega^{2} L^{2} N / s_{0} \leq 1 / 2$,

$$
\left\|\left(q_{1}, q_{2}\right)\right\|_{2} \leq 2 L^{2}\left\|\left(f_{1}, f_{2}\right)\right\|_{2}
$$

As $\left\|\left(f_{1}, f_{2}\right)\right\|_{2}=O\left(\omega^{2}\right)$, (3.11) implies $\left\|\left(q_{1}, q_{2}\right)\right\|_{2}=O\left(\omega^{2}\right)$ for $\omega \ll 1$. For $\omega^{2} \geq M=$ $M\left(N, m, s, r_{0}\right), M$ large enough, $\alpha \sim-\frac{N}{m}$, (3.9) shows:

$$
\left\|\left(q_{1}, q_{2}\right)\right\|_{2} \leq \frac{2 m}{N}\left\|\left(f_{1}, f_{2}\right)\right\|_{2}
$$

When $\omega_{0}^{2} \leq \omega^{2} \leq M,|\beta|$ is bounded from below uniformly in $\omega$ :

$$
|\beta| \geq \frac{N r_{0}|\omega|^{3}}{\left\|-m \omega^{2}+s(x)\right\|_{\infty}^{2}+r_{0}^{2} \omega^{2}} \geq \beta_{0},
$$


for some positive constant $\beta_{0}$ only depending on $r_{0}, m, L$, and $s(x)$. Inequality (3.8) gives:

$$
\left\|\left(q_{1}, q_{2}\right)\right\|_{2} \leq \beta_{0}^{-1}\left\|\left(f_{1}, f_{2}\right)\right\|_{2}=\beta_{0}^{-1} C_{1}\left(N, m, r_{0}, L, s\right)\left|P_{\text {in }}\right|
$$

for positive constant $C_{1}\left(N, m, r_{0}, L, s\right)$, uniformly in $\omega^{2} \in\left[\omega_{0}^{2}, M\right]$. Combining (3.11), (3.12), and (3.13), we see that for any $\omega$, and any given $P_{i n}$, there is a unique solution $P,\|P\|_{2} \leq C_{2}\left|P_{\text {in }}\right|$, for constant $C_{2}$ independent of $\omega$. The lemma is proved by applying the $L^{2}$ estimate and the $P$ equation (3.5).

\subsection{Nonlinear Waves}

We are interested in the persistence of multitone solutions when nonlinearities are present. For simplicity, we shall consider: (A1) $r_{a}>0$, a constant, and $P(u)=u^{2}$, the overall nonlinearity is cubic. As for linear waves, assume that (A2) $\epsilon_{s}=0, s(x) \geq s_{0}>0, s \in C^{1}([0, L])$. We prove:

Theorem 3.1 (Existence and Uniqueness of Multitone Solutions) Let the left boundary condition be:

$$
f_{i n}(t)=\sum_{j=1, \cdots, m} a_{j} \exp \left\{i \omega_{j} t\right\}+c . c .,
$$

and fix $\rho \geq 1$. Then under (A1-(A2) and for $\gamma$ small enough (independent of $\rho$ ), system (1.1)(1.2) has a unique solution of the form:

$$
u(x, t)=\sum_{k \in Z^{m}} U_{k}(x) \exp \{i k \cdot \omega t\}+c . c
$$

where $\omega=\left(\omega_{1}, \omega_{2}, \cdots, \omega_{m}\right)$, and complex valued functions $U_{k}(x) \in H^{1}([0, L])$, such that:

$$
\|u\| \equiv \sum_{k} \rho^{|k|}\left\|U_{k}\right\|_{H^{1}}<\infty
$$

The pressure $p$ is similar.

Proof: Let $B$ be the Banach space consisting of space-time functions of the form (3.14) with norm (3.15). Let $B_{1}=\left\{v \in B: v_{t} \in B\right\}$, a subspace of $B$. Consider the mapping $M: v \in B_{1} \rightarrow u$ defined as the unique bounded solution of the following equation in $B_{1}$ :

$$
\begin{aligned}
m u_{t t}+ & \int_{x}^{L} d x^{\prime} \int_{0}^{x^{\prime}} d x^{\prime \prime} N u_{t t}+f_{i n}(t)(L-x) \\
& +r_{a} u_{t}+s(x) u=-\gamma r_{n l}\left(x, v^{2}\right) v_{t}
\end{aligned}
$$

where $\gamma r_{n l}$ is the nonlinear nonlocal part of the damping function. 
Let us show that $M$ is a well-defined bounded mapping from $B_{1}$ to itself. First we notice that for any functions $u_{i} \in B, i=1,2$ :

$$
\begin{aligned}
u_{1} \cdot u_{2} & =\sum_{k_{1} \in Z^{m}} u_{1, k_{1}} e^{i k_{1} \cdot \omega t} \sum_{k_{2} \in Z^{m}} u_{2, k_{2}} e^{i k_{2} \cdot \omega t} \\
& =\sum_{j \in Z^{m}}\left(\sum_{k_{1}+k_{2}=j} u_{1, k_{1}} u_{2, k_{2}}\right) e^{i j \cdot \omega t}
\end{aligned}
$$

so:

$$
\begin{aligned}
\left\|u_{1} u_{2}\right\|= & \sum_{j \in Z^{m}} \rho^{|j|}\left\|\sum_{k_{1}+k_{2}=j} u_{k_{1}} u_{k_{2}}\right\|_{H^{1}} \\
& \leq \sum_{k_{1}, k_{2} \in Z^{m}} \rho^{\left|k_{1}\right|+\left|k_{2}\right|}\left\|u_{k_{1}} u_{k_{2}}\right\|_{H^{1}} \\
& =\sum_{k_{1} \in Z^{m}} \rho^{\left|k_{1}\right|}\left\|u_{k_{1}}\right\|_{H^{1}} \sum_{k_{2} \in Z^{m}} \rho^{\left|k_{2}\right|}\left\|u_{k_{2}}\right\|_{H^{1}}=\left\|u_{1}\right\|\left\|u_{2}\right\| .
\end{aligned}
$$

It follows that:

$$
\left\|r_{n l}\left(x, v^{2}\right) v_{t}\right\| \leq C\left\|v^{2} * K\right\|\left\|v_{t}\right\| \leq C^{\prime}\left\|v^{2}\right\|\left\|v_{t}\right\|=C^{\prime}\|v\|^{2}\left\|v_{t}\right\|
$$

where $C^{\prime}$ depends on the kernel function $K$, and $*$ denotes the convolution integral on $[0, L]$. Denoting $F(x, t)=-\gamma r_{n l}\left(x, v^{2}\right) v_{t} \in B$, we show that $u \in B_{1}$. Write $F(x, t)=\sum_{k \in Z^{m}} F_{k} e^{i k \cdot \omega t}$ + c.c, then (3.16) is same as the system:

$$
\begin{aligned}
p_{x x} & -N u_{t t}=0 \\
p & =m u_{t t}+r_{a} u_{t}+s(x) u+F
\end{aligned}
$$

with boundary condition: $p_{x}(0, t)=f_{\text {in }}(t), p(L, t)=0$. Seek solution of system (3.19) in the form:

$$
\begin{aligned}
& p=\sum_{k \in Z^{m}} p_{k} e^{i k \cdot \omega t}+c . c, \\
& u=\sum_{k \in Z^{m}} u_{k} e^{i k \cdot \omega t}+c . c,
\end{aligned}
$$

resulting in $\left(k=\left(k_{1}, k_{2}, \cdots, k_{m}\right)\right)$ :

$$
\begin{array}{r}
p_{k}=\left(-m(k \cdot \omega)^{2}+i r_{a}(k \cdot \omega)+s(x)\right) u_{k}+F_{k}, \\
p_{k, x x}+N(k \cdot \omega)^{2} u_{k}=0,
\end{array}
$$

with boundary condition: $p_{k}(L)=0, p_{k, x}(0)=0$ if $k$ is not one of the $m$ modes (along $e_{j}$, $j=1,2, \cdots, m)$ of $f_{i n}$; otherwise $p_{k, x}(0)=a_{j}$, if $k=e_{j}$. 
For each $k$, the system (3.20) can be uniquely solved as in Lemma 4.1, with the estimates:

$$
\begin{aligned}
\left\|p_{k}\right\|_{H^{1}} & \leq C_{1}\left\|F_{k}\right\|_{2}+C_{2}\left\|f_{i n, k}\right\|_{2}, \\
\left\|u_{k}\right\|_{H^{1}} & \leq C_{3}\left\|p_{k}\right\|_{H^{1}} /(1+|k \cdot \omega|) .
\end{aligned}
$$

It follows that the mapping $M$ is from $B_{1}$ to itself, and it is not hard to check that $M$ is a contraction mapping if $\gamma$ is small enough. The proof is done.

\section{Numerical Results}

The model system is computed with a second order semi-implicit finite difference method, we refer to [21] for its details and choice of the coefficient functions in the model other than $P(u)=u^{2}$. The input at the left boundary $x=0$ is the sum of two tones (sinusoids) at frequencies $f_{1}=3.5 \mathrm{kHz}$ (kilo Hertz) and $f_{2}=4 \mathrm{kHz}$, with amplitudes $80 \mathrm{~dB}$ (decibel) and $85 \mathrm{~dB}$ respectively. The zero decible is $20 \mu \mathrm{Pa}$ in physical unit. The time step is $0.01 \mathrm{~ms}$ (millisecond), and spatial grid is $0.01 \mathrm{~cm}$. The computation ends at $20 \mathrm{~ms}$ when the BM responses reach a steadily oscillating state. To observe the frequency content of such a state, we select four points $\left(x_{j}\right.$ 's, $\left.j=1,2,3,4\right)$ on BM, and examine the response time series $\left(u\left(x_{j}, t\right)\right)$ at these points from $5 \mathrm{~ms}$ to $20 \mathrm{~ms}$ (to omit initial transient effects). The power spectral density of $u\left(x_{j}, t\right)$ at each $j$ is obtained using signal processing tool (sptool) in Matlab, to illustrate the energy distribution across frequencies in log-scale.

Figure (11) top frame shows the time series of BM displacement at $x=1.93 \mathrm{~cm}$, the bottom frame is the log-log plot of FFT power spectral density vs. frequency. We see the major peak at $3.5 \mathrm{kHz}$, as $x=1.93 \mathrm{~cm}$ is the (so called characteristic) location for the peak of a single $3.5 \mathrm{kHz}$ tone. In addition, we see two small side peaks at $3 \mathrm{kHz}\left(=2 f_{1}-f_{2}\right)$, and $4 \mathrm{kHz}\left(f_{2}\right)$. In figure (21), at $x=1.85 \mathrm{~cm}$, the characteristic location for $f_{2}$, the $f_{2}$ peak is more pronounced, however

the $f_{1}$ peak is still the highest. Such effect of lower frequency tone $f_{1}$ to a higher frequency tone $f_{2}$ is called upward masking in hearing. In addition, there are two small side peaks at $3.0 \mathrm{kHz}$ $\left(=2 f_{1}-f_{2}\right)$ and $4.5 \mathrm{kHz}\left(=2 f_{2}-f_{1}\right)$. In figure (3), at $x=2.03$, the characteristic location for $3 \mathrm{kHz}$, a dominant single peak due to the generated combination tone $2 f_{1}-f_{2}$ is observed. In contrast, the $2 f_{2}-f_{1}$ tone $(=4.5 \mathrm{kHz})$ is weaker and dominated by $f_{1}$ and $f_{2}$ even at its characteristic location $x=1.69 \mathrm{~cm}$, see figure (44). The above findings on combination tones are consistent with the experiments on cochlea [17, and the analytic structures of multi-tone solutions in the previous section. 


\section{Conclusions}

The nonlinear nonlocal cochlear models of the transmission line type are well-posed globally in time and admit exact multi-frequency solutions in the weakly cubic nonlinear regime. For finitely many tonal input at distinct frequencies, the exact solutions contain all integral linear combinations of input frequencies. For two tone input with frequencies $f_{1}$ and $f_{2}$ at high enough intensities, we observed numerically the combination tones $2 f_{1}-f_{2}$ and $2 f_{2}-f_{1}$ in model output, in agreement with existing experimental observations [17] and the structure of analytical solutions.

\section{Acknowledgements}

J. X. would like to thank P. Collet for a stimulating discussion of quasiperiodic solutions in dissipative systems, and a visiting professorship at the Inst. H. Poincaré, where the work was in progress. He also thanks H. Berestycki, P. Constantin, G. Papanicolaou, and J-M Roquejoffre for their interest.

Part of the work was done while Y.Q. was a TICAM (Texas Institute of Computational and Applied Math) visiting fellow at the University of Texas at Austin. The TICAM research fellowship is gratefully acknowledged.

\section{References}

[1] Allen, J. B., Cochlear Modeling-1980, in Lecture Notes in Biomathematics, Springer-Verlag, 1980, eds. M. Holmes and L. Rubenfeld, volume 43, pp 1-8.

[2] de Boer, E., Mechanics of the Cochlea: Modeling Efforts, in Springer Handbook of Auditory Research, Springer-Verlag, 1996, eds. P. Dollas and A. Popper and R. Fay, volume 8, pp $258-317$.

[3] L. Deng, Processing of acoustic signals in a cochlear model incorporating laterally coupled suppresive elements, Neural Networks, 5(1), pp 19-34, 1992.

[4] Geisler, C. D., From Sound to Synapse, Oxford University Press, Oxford, 1998.

[5] J. J. Guinan and W. T. Peake, Middle-Ear Characteristics of Anesthesized Cats, J. Acoust. Soc. Amer, 41(5), pp 1237-1261, 1967. 
[6] J. L. Hall, Two-tone suppression in a nonlinear model of the basilar membrane, J. Acoust. Soc. Amer, volume 61, pp 802-810, 1977.

[7] Y. Jau and C. D. Geisler, Results from a cochlear model utilizing longitudinal coupling, in Mechanics of Hearing, Martinus Nijhoff Pub., Delft Univ. Press, 1983, E. de Boer and M. Viergever, pp 169-176.

[8] J. B. Keller, J. C. Neu, Asymptotic analysis of a viscous cochlear model, J. Acoust. Soc. America, 77(6), pp 2107-2110, 1985.

[9] D. O. Kim, An overview of nonlinear and active models, in Lecture Notes in Biomathematics: Peripheral Auditory Mechanisms, Springer-Verlag, 1986, eds J. Allen and J.L. Hall and A. Hubbard and S.T. Neely and A. Tubis, volume 64, pp 239-249.

[10] R. Leveque, Ch. Peskin, P. Lax, Solution of a Two-Dimensional Cochlear Model with Fluid Viscosity, SIAM J. Applied Math, v. 48, no. 1, pp 191-213, 1988.

[11] M. C. Liberman, The cochlear frequency map for the cat: Labeling auditory nerve fibers of known characteristic frequency, J. Acoust. Soc. Amer, volume 72, pp 1441-1449, 1982.

[12] R. Lyon, A computational model of filtering, detection, and compression in the cochlear, IEEE International Conference on Acoustics, Speech and Signal Processing, pp 1282-1285, 1982.

[13] R. Meddis, L. O'Mard, E. Lopez-Poveda, A computational algorithm for computing nonlinear auditory frequency selectivity, J. Acoust. Soc. America 109(6), 2001, pp 2852-2861.

[14] S. Neely, Mathematical modeling of cochlear mechanics, J. Acoust. Soc. Amer, 78(1), pp 345-352, 1985.

[15] S. Osher, L. Rudin, Feature-Oriented Image Enhancement Using Shock Filters, SIAM J. Numer. Analysis, Vol. 27, No. 4, pp 919-940, 1990.

[16] K. Pohlmann, Principles of Digital Audio, 4th edition, McGraw-Hill Video/Audio Professional, 2000.

[17] L. Robles and M. Ruggero, Mechanics of the Mammalian Cochlea, Physiological Reviews, 81(3), pp 1305-1352, 2001. 
[18] Sondhi, M., The Acoustical Inverse Problem for the Cochlea, Lecture Notes in Biomathematics, Springer-Verlag, 1980, eds M. Holmes and L. Rubenfeld, volume 43, pp 95-104.

[19] H. W. Strube, A Computationally Efficient Basilar-Membrane Model, Acustica, volume 58, pp 207-214, 1985.

[20] von Békésy, G., Experiments in Hearing, McGraw-Hill, New York, 1960.

[21] J. Xin, Y-Y Qi, L. Deng, Time Domain Computation of a Nonlinear Nonlocal Cochlear Model with Applications to Multitone Interaction in Hearing, Communications in Math Sciences, Vol. 1, No. 2, pp 211-227, 2003 (www.intlpress.com/CMS).

[22] E. Zeidler, Applied Functional Analysis: Main Principles And Their Applications, New York, 1995. 

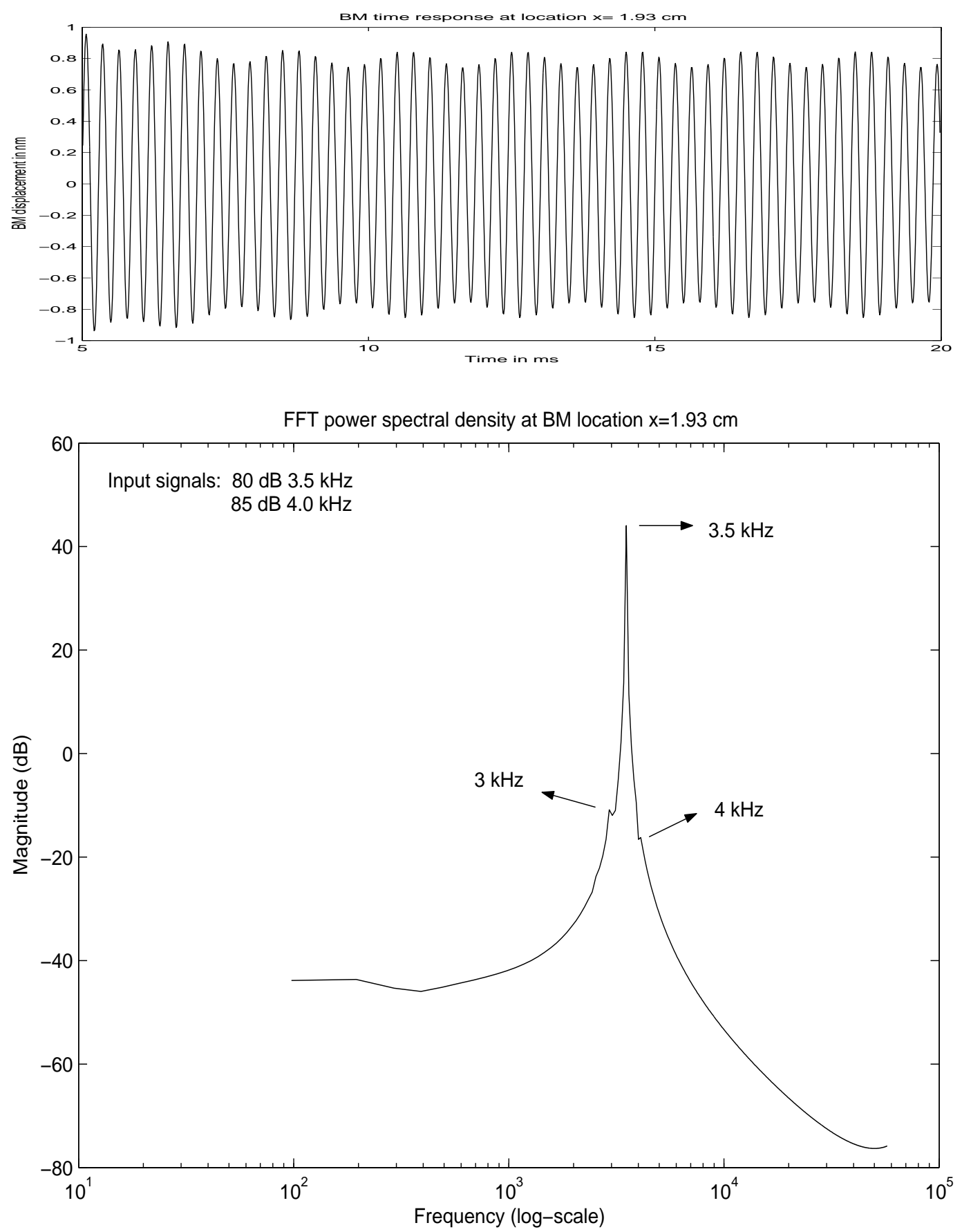

Figure 1: Time series of BM displacement at $x=1.93 \mathrm{~cm}$ (top frame), and its FFT power spectral density vs. frequency (bottom frame). 

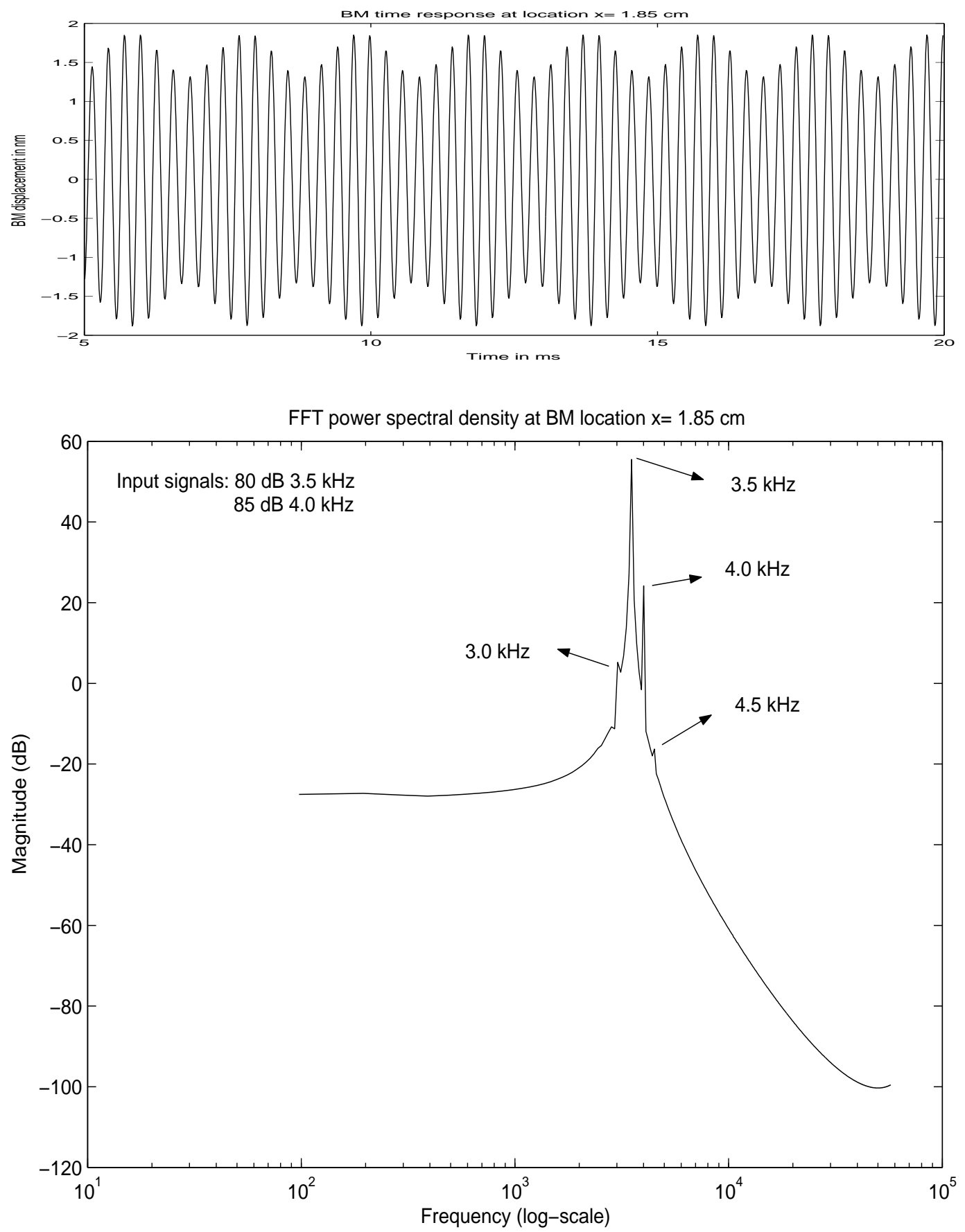

Figure 2: Time series of BM displacement at $x=1.85 \mathrm{~cm}$ (top frame), and its FFT power spectral density vs. frequency (bottom frame). 

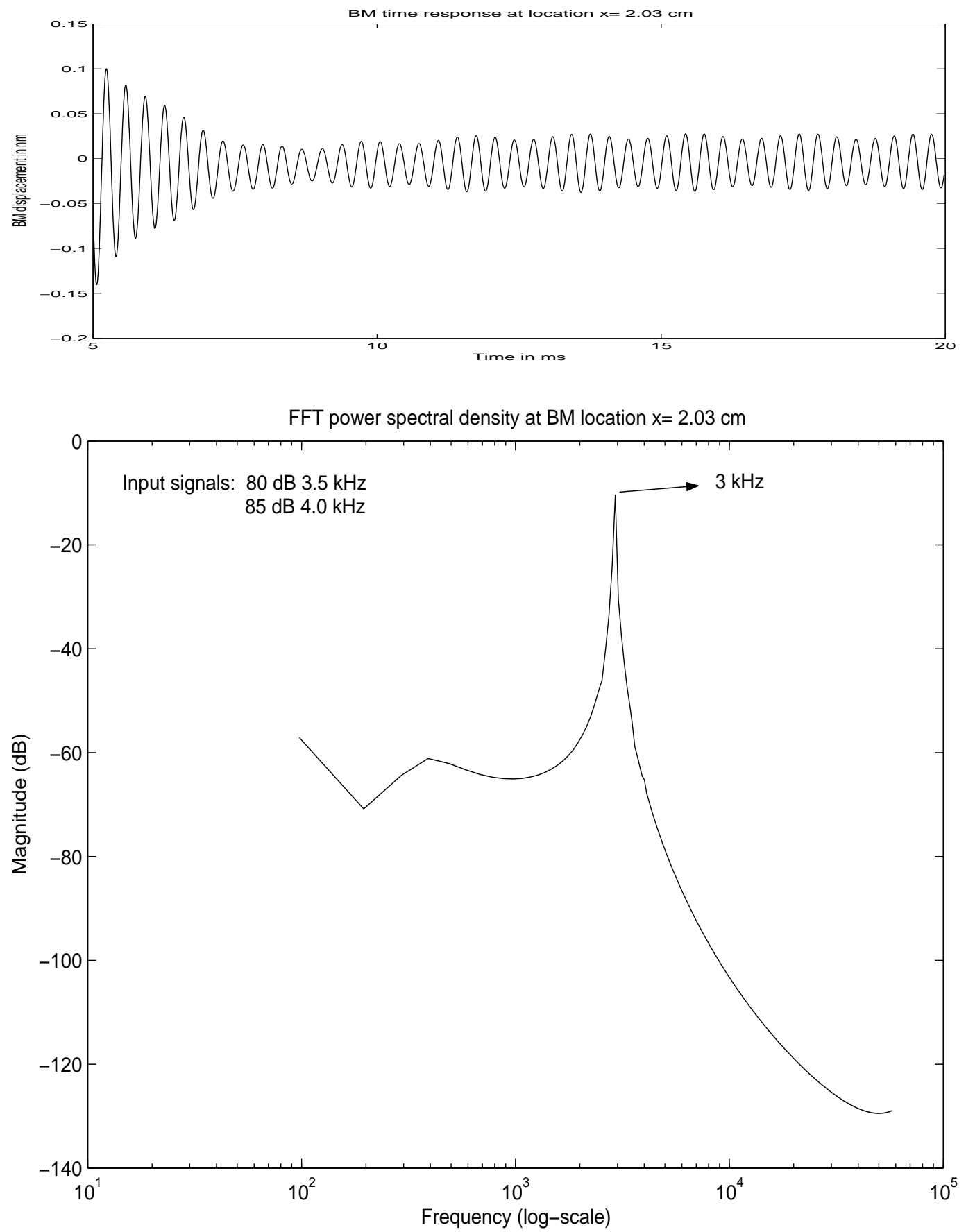

Figure 3: Time series of BM displacement at $x=2.03 \mathrm{~cm}$ (top frame), and its FFT power spectral density vs. frequency (bottom frame). 

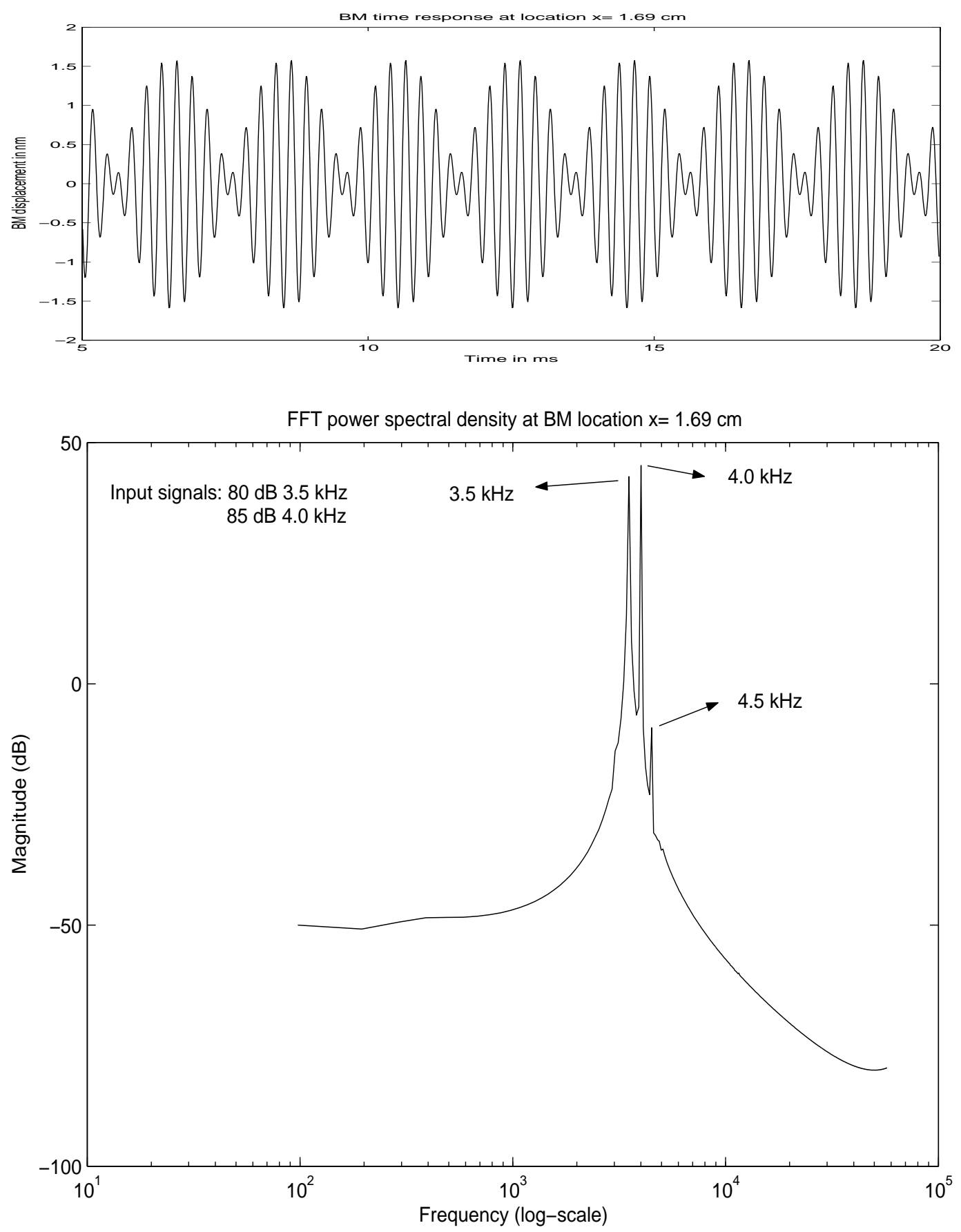

Figure 4: Time series of BM displacement at $x=1.69 \mathrm{~cm}$ (top frame), and its FFT power spectral density vs. frequency (bottom frame). 